\title{
Enseñar filosofía a través del cine: un panorama bibliográfico
}

Lauro Zavala ${ }^{1 \oplus}$

Universidad Autónoma Metropolitana - Xochimilco, México

Autor de correspondencia: ${ }^{1}$ zavala38@hotmail.com Recibido: 9 de noviembre de 2020 Revisado: 13 de noviembre de 2020 Aprobado: 6 de abril de 2021 Publicado: 4 de mayo

\section{Resumen}

En esta revisión bibliográfica de carácter panorámico sobre cine y filosofía, se observa una distinción notable entre la filosofía continental y la filosofía analítica. En la tradición continental, numerosos pensadores contemporáneos han dedicado sus más recientes estudios a pensar el cine, especialmente después del trabajo seminal de Gilles Deleuze. Por otra parte, en la tradición analítica existe una corriente de pensamiento filosófico con vocación pedagógica - en concordancia con la vocación pedagógica del cine mismo-, desde la cual se estudian películas, directores y géneros como herramientas para la enseñanza de lógica, estética, ética, bioética, ética médica, ética ambiental y política. Desde esta perspectiva, se pretende aprender filosofía al mismo tiempo que se aprecian los alcances trascendentes de la experiencia cinematográfica durante y después de la proyección. En este trabajo se exploran las posibilidades pedagógicas de ambas tradiciones y se enfatiza en los alcances de la tradición analítica, por su interés para la enseñanza de la filosofía $-o$ de cualquier otra disciplina - a través del cine.

Palabras clave: cine, filosofía continental, filosofía analítica, educación, análisis 


\title{
Teaching philosophy through film: a bibliographical overview
}

\begin{abstract}
In this panoramic bibliographical review of film and philosophy, there is a remarkable distinction between continental philosophy and analytical philosophy. In the continental tradition, several contemporary scholars have devoted their most recent studies to thinking about film, especially after the pioneering work of Gilles Deleuze. On the other hand, in the analytical tradition, there is a school of philosophical thought with a pedagogical vocationin line with the pedagogical vocation of film itself-from which movies, directors, and genres are studied as tools for the teaching of logic, aesthetics, ethics, bioethics, medical ethics, environmental ethics, and politics. From this perspective, the aim is to learn philosophy while appreciating the transcendent scope of the cinematographic experience during and after the screening. This article explores the pedagogical possibilities of both traditions and emphasizes the scope of the analytical tradition, given its relevance for the teaching of philosophy-or any other discipline-through film.
\end{abstract}

Keywords: cinema, continental philosophy, analytic philosophy, education, analysis

\section{O ensino da filosofia através do cinema: um panorama bibliográfico}

\section{Resumo}

Nesta revisão bibliográfica panorâmica do cinema e da filosofia, há uma distinção notável entre filosofia continental e filosofia analítica. Na tradição continental, vários estudiosos contemporâneos têm dedicado seus estudos mais recentes ao pensamento sobre cinema, especialmente após o trabalho pioneiro de Gilles Deleuze. Por outro lado, na tradição analítica, existe uma corrente de pensamento filosófico com vocação pedagógica conforme a vocação pedagógica do próprio cinema - da qual se estudam filmes, diretores e gêneros como ferramentas para o ensino da lógica, da estética, da ética, da bioética, da ética médica, da ética ambiental e da política. Nessa perspectiva, o objetivo é aprender filosofia enquanto se aprecia o alcance transcendente da experiência cinematográfica durante e após a projeção. Este artigo explora as possibilidades pedagógicas de ambas as tradições e enfatiza o alcance da tradição analítica, dada sua relevância para o ensino da filosofia - ou qualquer outra disciplina - através do cinema.

Palavras-chave: cinema, filosofia continental, filosofia analítica, educação, análise 
Desde su nacimiento, el cine ha ejercido una poderosa atracción sobre el pensamiento filosófico. En este trabajo se presenta una revisión bibliográfica sobre el estado actual del diálogo entre filosofía y cine en varios terrenos de la enseñanza universitaria y preuniversitaria. En términos generales, es posible establecer una distinción muy notable entre la tradición de la filosofía continental y la tradición de la filosofía analítica (D’Agostini, 2000).

La filosofía continental - llamada así por surgir en el corazón del continente europeo, especialmente en Alemania y Francia-, inicia con Kant, Nietzsche, Hegel y Heidegger, y llega hasta Foucault, Derrida y Zizek (Schrift, 2010; Solomon \& Sherman, 2009). Se caracteriza por su naturaleza especulativa e historiográfica. Incluye el idealismo alemán, el marxismo, la fenomenología, el existencialismo, la hermenéutica, el estructuralismo y la deconstrucción. En gran medida, esta tradición filosófica comparte la tesis kantiana que sostiene que la reflexión filosófica es el sustento de todo conocimiento - en lugar del estudio empírico de casos particulares- (Mullarkey \& Lord, 2009). Esta tradición filosófica tiende a ser poco accesible a un público no especializado. Al mismo tiempo, tiene una presencia muy notable en la región iberoamericana, en particular debido a las traducciones hechas en España de numerosos textos filosóficos escritos en francés. De ahí se desprende que la filosofía escrita en francés - sobre todo continental - tenga una presencia marcada en la tradición filosófica hispanoamericana.

En contraste, la filosofía analítica tiene su origen en el análisis del lenguaje por medio de la lógica formal. Inicia en el mundo anglosajón - Inglaterra y Estados Unidos- con el trabajo de Bertrand Russell, Gottlob Frege, Karl Popper y el primer Wittgenstein, y llega hasta Thomas Kuhn, Mario Bunge, Paul Feyerabend y Richard Rorty (Glock, 2008; Sorell \& Rogers, 2005). En la segunda mitad del siglo XX, la tradición analítica se expande hasta cubrir la exigencia de claridad en la argumentación lógica, el respeto a las ciencias naturales y exactas y la desconfianza frente a los grandes sistemas filosóficos, en particular, frente a la metafísica. El interés central de la filosofía analítica es la precisión argumentativa, en oposición a los grandes sistemas de pensamiento y las posibles recetas para saber cómo vivir (Beaney, 2017; Dainton \& Robinson, 2014).

En la tradición continental, numerosos pensadores contemporáneos han dedicado sus más recientes estudios a pensar el cine, especialmente después del trabajo seminal de Gilles Deleuze (1981; 1983; 1985), como es el caso de Jacques Rancière (2001; 2010; 2011; 2014), Jean-Luc Nancy, Georges Didi-Huberman (2016) y muchos otros (Sinnerbrink, 2011). Por otra parte, en la tradición analítica existe una corriente de pensamiento filosófico con vocación pedagógica —en concordancia con la vocación pedagógica del cine mismo-, desde la cual se utiliza el estudio de películas, directores y géneros como herramientas para la enseñanza de lógica, estética, ética, bioética, ética médica, ética ambiental y política. Desde esta perspectiva, se pretende aprender filosofía al mismo tiempo que se aprecian los alcances trascendentes de la experiencia cinematográfica durante y después de la proyección.

Ambas tradiciones filosóficas - continental y analítica - tienen consecuencias muy específicas en el salón de clases. Mientras la tradición continental propicia encuadrar un curso y una clase particular desde el marco teórico para después - si hay tiempo- aplicar estos conceptos al estudio de casos, la tradición analítica propicia iniciar una clase o un 
curso con la presentación de un caso particular, a partir del cual se podrán mostrar los fundamentos teóricos y conceptuales que explican ese y muchos otros casos.

En Latinoamérica hay una mayor familiaridad con la tradición continental, pero es necesario reconocer que la tradición analítica, al ser casuística, perspectivista y narrativa, tiene una mayor efectividad en el terreno pedagógico. Los principios de la filosofía continental se pueden observar en el salón de clases, donde se suele partir de las teorías para acceder más adelante al estudio de casos. Ésta es una tradición centrada en los textos escritos, en las definiciones generales y en los grandes sistemas de pensamiento. Por su parte, las prácticas docentes sustentadas en la tradición analítica utilizan los recursos de la imagen, inician con el examen de casos específicos — tradición casuística- y enfatizan la coherencia argumentativa.

En la primera sección de este trabajo presento algunos rasgos de la tradición continental en el empleo del cine para hacer filosofía. En las secciones siguientes, por su interés para la pedagogía, doy cuenta de los terrenos que están estudiando el cine con los recursos de la filosofía analítica.

\section{El cine como máquina para pensar}

En la tradición continental de los estudios sobre cine, numerosos filósofos utilizan el cine y su historia como una oportunidad para pensar el mundo y para plantear problemas filosóficos vinculados al tiempo, a la realidad, a la percepción y al movimiento, entre otros objetos de la investigación filosófica.

Es notable que la mayor parte de estos filósofos sean de origen francés y se hayan aproximado al cine al acercarse al final de su carrera filosófica. Incluso, algunos de ellos -como Clément Rosset - vieron su primera película al terminar su adolescencia (Rosset, 2010, p. 7). En ese sentido, el cine no forma parte de su formación personal desde la infancia. En sus propias palabras, no son espectadores cinéfilos, sino que se acercan al cine por considerar que ofrece la oportunidad de plantear problemas filosóficos (Bernini, Gaetano \& Dottorini, 2015) y, como señala Chateau (2005), al descubrir que el cine es un material atractivo, flexible y trascendente.

Éste es el caso de la mayoría de los filósofos de formación continental, que trabajan en la tradición deductivista y nomotética. Se trata de pensadores como Gilles Deleuze, Jacques Ranciere, Jean-Luc Nancy, Julia Kristeva, Georges Didi-Huberman, Alan Badiou, Jean-Luc Comolli, Pascal Bonitzer, Clément Rosset, Gilles Lipovetsky, Jacques Derrida, Paul Virilio, Jean Baudrillard, Felix Guattari y Giorgio Agamben. Todos ellos escriben originalmente en francés - lo que ha garantizado su traducción al español-. También es necesario añadir a Stanley Cavell y Slavoj Zizek, que escriben originalmente en inglés.

Todos ellos continúan una tradición filosófica que se inicia con Sigfried Kracauer, Theodor Adorno -sociólogos alemanes-, Henri Bergson, Maurice Merleau-Ponty, Emmanuel Levinas - filósofos franceses- y algunos críticos y escritores como Antonin Artaud, André Bazin y Roland Barthes - también franceses- (Colman, 2009). Por otra parte, se deben también tener en cuenta algunos teóricos del cine como Hugo Munsterberg -estadounidense-, Serge Daney, Jean-Louis Baudry, Christian Metz -franceses-, Stephen Heath, Laura Mulvey, Daniel Frampton -ingleses- y el director francés más 
filosófico de la historia del cine: Jean-Luc Godard.

Al llegar a este punto, es necesario reconocer que las propuestas filosóficas de todos estos pensadores son plataformas para pensar, pero no tienen aplicabilidad en el momento de analizar una secuencia particular. Para analizar secuencias de cine es necesario, paradójicamente, buscar las categorías filosóficas desarrolladas en las obras de estos pensadores en las que no han pensado el cine. Así, por ejemplo, como se demuestra en el trabajo de Esquenazi (2017), ninguna de las numerosas categorías filosóficas propuestas por Gilles Deleuze en sus libros sobre cine es útil para analizar una secuencia. Para ello, en cambio, es necesario utilizar categorías deleuzianas tales como la territorialización, el pliegue y la bifurcación, provenientes de Mil mesetas (1972), El pliegue (1989) y Diferencia y repetición (2002), respectivamente. Algo similar ocurre con los otros filósofos que consideran al cine como una máquina para pensar.

En esta tradición filosófica se tiende a privilegiar el principio de autoridad, donde es central entender quién dijo qué, es decir, qué filósofo del canon pensó tal o cual cosa. Se descalifica como una filosofía salvaje a la filosofía que privilegia la formulación de preguntas, el empleo de métodos sistemáticos de argumentación lógica y la vocación didáctica en particular en el análisis de secuencias-. Debido a que el interés de este trabajo está orientado al estudio de los métodos de análisis de carácter pedagógico, en lo que sigue se dará mayor peso al estudio de la tradición analítica en los estudios sobre cine.

\section{La filosofía al servicio del cine}

En contraste con la tradición académica continental, los filósofos que se presentan a continuación pertenecen a una tradición distinta en la forma de hacer filosofía. Se trata de la tradición analítica, casuística y humanística, que en gran medida es de origen anglosajón $-\mathrm{y}$ en esa medida, es una tradición casi totalmente desconocida en la región latinoamericana, pues sus materiales no suelen ser traducidos al español-. Se trata de filósofos que parten de una cinefilia iniciada desde la infancia y que consideran el cine, antes que nada, como una experiencia emocional y corporal, en la que el espectador se sumerge y de la cual sale transformado.

Esta tradición filosófica constructivista, con frecuencia vinculada a las teorías cognitivas del cine, es compatible con el feminismo actual y muy próxima a las neurociencias experimentales. En esta tradición, el cine es "el invento más sofisticado creado por el hombre para simular y manipular los diferentes aspectos de las maneras como percibimos, sentimos, pensamos, actuamos, memorizamos, asociamos y socializamos" (Grodal, 1997, p. 278), al considerar su natural potencial de pedagogía social. Así, el cine nos puede enseñar a ser más conscientes de nuestra condición como parte de la naturaleza, lo que significa que en esta tradición se otorga un lugar central a la experiencia estética y a los contenidos éticos de las películas.

Desde esta perspectiva, el cine no es una maquinaria para pensar el mundo, sino una experiencia que puede ser aprovechada para aprender a razonar, a argumentar, a convivir con los demás y a entender las posibilidades y los límites de la naturaleza, de la sociedad y de la persona. La filosofía, entonces, puede estar al servicio del cine, al estudiar su naturaleza como una experiencia única, a la vez contingente y trascendente, irrepetible y compartida. 
Asimismo, ver una película es una experiencia educativa por definición. El cine puede ser utilizado para aprender y enseñar filosofía - o cualquier otra disciplina del conocimiento-. Es una herramienta de enseñanza por su propia naturaleza pedagógica.

Hablamos de teóricos del cine como Noël Carroll, Gregory Currie, David Bordwell, Carl Plantinga, Richard Allen, Edward Branigan, Stephen Prince, Brian Henderson, V. F. Perkins, Peter Wollen, D. N. Rodowick, Robert Stam, Cynthia Freeland, Ian Jarvie, Torben Grodal, Thomas Elsaesser, Annette Insdorf y Thomas Wartenberg. Todos ellos escriben originalmente en inglés. A su trabajo habría que añadir el de otros analistas, como Jacques Aumont, Michel Marie, Laurent Jullier - franceses-, Werner Faulstich, Helmut Korte, Königs Lernhilfen, Alice Bienk — de Alemania y Suiza - y Francesco Casetti -italiano-.

Estos autores continúan una tradición en el estudio del lenguaje cinematográfico iniciada por Bela Balász —en Hungría-, Rudolf Arnheim —en Alemania-, John Fell y Seymour Chatman - en Estados Unidos- y Daniel Arijon - en Francia-. A esta tradición formalista pertenecen los autores de los libros de texto para el análisis de la forma fílmica, como Lincoln F. Johnson —años 60-, James F. Scott —años 70-, Bernard K. Dick —años 80-, Lee R. Bobker —años 90-, Joseph M. Boggs —años 2000- y, más recientemente, Louis Gianetti, Michael Ryan, David Barsam —en Estados Unidos-, Timothy Corrigan, Patricia White y Warren Buckland - en Inglaterra-. Por cierto, en la región iberoamericana ya hay algunos teóricos y analistas del cine - aún ningún editor ha traducido sus trabajos al inglés-. Entre ellos es posible mencionar a Jesús González Requena, Santos Zunzunegui y Javier Marzal Felici —en España-, Ángel Faretta —en Argentina-, Oswaldo Osorio —en Colombia- y Álvaro Fernández —en México-.

En esta tradición filosófica y analítica se tiende a privilegiar la formulación de preguntas por sobre el principio de autoridad. Es importante pensar de nuevo y replantear en distintos contextos los problemas de la lógica, de la epistemología, de la ética y de la estética. Esta forma de hacer filosofía no es ajena a las polémicas disciplinarias. Es así como se suele descalificar a esta filosofía, que privilegia el estudio de autores y textos particulares, como una filosofía escolar.

En lo que sigue presento algunos de los trabajos más recientes que forman parte de esta tradición académica, elaborados desde la perspectiva analítica.

\section{Enseñanza de la filosofía a través del estudio de películas}

En esta tradición hay tres vertientes: la primera utiliza las películas para estudiar la historia de la filosofía; la segunda utiliza las ideas de los grandes filósofos para entender la naturaleza de películas particulares; y la tercera consiste en estudiar una película - o una serie de televisión - desde una perspectiva filosófica particular.

En la primera vertiente encontramos el trabajo de Falzon. Su libro La filosofía va al cine (2005) está organizado a partir de los grandes problemas de la ética, de la estética, de la ontología, de la epistemología y de la lógica -en ese orden-. La discusión de estos problemas se ilustra con la recreación de escenas y diálogos tomados de diversas películas. Por ejemplo, en el capítulo final -El Santo Grial- se explican los principios básicos de la lógica argumentativa a partir de algunos diálogos absurdos de Los caballeros de la mesa 
cuadrada (Gilliam, 1975), del grupo Monthy Python y se ejemplifican los tipos de falacias posibles a partir de escenas tomadas de películas de Stanley Kubrick -Cómo aprendí a amar la bomba (1964)_, Francis Ford Coppola - Apocalipsis ahora (1979)—, Woody Allen -Interiores (1978) - y Alfred Hitchcock — La ventana indiscreta (1954)_, entre otros directores. Veamos por un momento el método de Falzon (2005) al utilizar escenas de películas canónicas para explicar los principios de la argumentación lógica. Este es un pasaje de la sección dedicada a explicar las falacias formales:

Entre las falacias que resultan de la forma del argumento (las falacias formales) se encuentran las dos conocidas como afirmar el consecuente y negar el antecedente.

En la falacia de afirmar el consecuente presentamos un argumento que se parece a la forma válida del Modus Ponens: "Si p entonces q; p; luego q". Sin embargo, al afirmar el consecuente vamos hacia atrás y argumentamos "Si p entonces q; q, luego p". Un ejemplo de esto aparece en La ventana indiscreta (Rear Window, Alfred Hitchcock, 1954). L. B. Jefferies (James Stewart) ha estado observando a su vecino, de quien sospecha que puede haber cometido un asesinato por el hecho de envolver en un periódico un cuchillo de carnicero y una pequeña sierra. Se lo cuenta a su novia Lisa (Grace Kelly), añadiendo que existe "algo terriblemente malo". Se imagina que esto demuestra efectivamente sus sospechas sobre el vecino. Sin embargo, pensar así significa caer en la falacia de afirmar el consecuente.

En la falacia de negar el antecedente tenemos un argumento parecido a la forma válida del Modus Tollens: "Si p entonces q; no q; luego no p". pero en lugar de eso estamos argumentando: "Si p entonces q; no p, luego no q". La falacia de negar el antecedente aparece en Interiores (Interiors, Woody Allen, 1978), cuando Pearl (Maureen Stapleton) dice en cierto momento: "Vivirás hasta los cien si renuncias a todas las cosas que te hacen desearlo". [...]. Renunciar a cosas agradables como fumar, beber y tener relaciones sexuales solo significa que no morirás a causa de ellas, pero existen otras muchas causas de muerte prematura, como, por ejemplo, que te atropelle un autobús. (Falzon, 2005, pp. 200-201).

La segunda modalidad en el empleo de películas para explicar ideas filosóficas consiste en estudiar una película particular desde diversas aproximaciones filosóficas. Por ejemplo, en un título que alude al pensamiento cartesiano, Terminator and Philosophy. I'll be Back, Therefore I Am [Terminator y la filosofía. Volveré, y por lo tanto existo], Brown et al. (2009) convocaron a varios filósofos profesionales a pensar Terminator (Cameron, 1984) desde perspectivas como el feminismo, la revolución marxista, las lenguas naturales y el autosacrificio. Esta estrategia didáctica es similar a la del conocido volumen colectivo Los Simpson y la filosofía (Irwin et al., 2009), donde se discute, entre otros temas, cuál es el valor heurístico de Bart o cómo adoptar una perspectiva kantiana para entender el universo moral de la familia Simpson. Otro caso similar es el del volumen colectivo La filosofía de House (Irwin \& Jacoby, 2009), donde se propone estudiar la serie Dr. House desde la lógica de las conjeturas y desde una perspectiva sartreana o nietzscheana. En Inglaterra, la prestigiosa editorial académica Routledge ha creado una serie a partir de esta idea, donde ya se han publicado volúmenes colectivos sobre la dimensión filosófica de películas como La delgada línea roja (Davies, 2009); Hable con ella (Eaton, 2009); Mulholland Drive (Giannopoulos, 2013); Eterno resplandor de una mente sin recuerdos (Grau, 2009); Memento (Kania, 2009) y muchas otras. 
La tercera estrategia en la que se utiliza el cine como herramienta para propiciar el pensamiento filosófico consiste en proponer una historia de la filosofía y detenerse en películas particulares. Este es el caso de Primum videre, deindre philosophare (Ferrer et al., 2006) y Cine: 100 años de filosofía. Una introducción a la filosofía a través del análisis de películas (Cabrera, 2015), ambos escritos en España. En el primero se seleccionan 17 filósofos, cuyas ideas son explicadas a partir de otras tantas películas. Así, por ejemplo, se explica el pensamiento de Platón a partir de La rosa púrpura del Cairo (Allen, 1985); el de Ockham a partir de El nombre de la rosa (Annaud, 1984); y el de Foucault a partir de Amélie (Jeunet, 2001).

En el libro de Cabrera (2015), se proponen 14 ejercicios filosóficos, en cada uno de los cuales se articula el pensamiento de un filósofo con una película particular - y después con otras películas similares-, con el fin de plantear un problema filosófico. Por ejemplo, en el ejercicio 4 se presentan las ideas de Francis Bacon y los presocráticos acerca del hombre y su relación con la naturaleza, para después estudiar las actitudes frente a la naturaleza contenidas en dos películas de Spielberg: Tiburón (1975) y Parque Jurásico (1993). Finalmente, en la tercera sección de este ejercicio se comentan algunas películas del llamado Cine de Catástrofes, donde se muestran las formas en las que el ser humano se relaciona con la naturaleza - ya sea como aparente dueño de los recursos naturales o como parte del ambiente natural-. En el ejercicio 7 se presentan las ideas de Kant sobre la moral, para después estudiar su presencia en la película Un hombre para la eternidad (Zimmermann, 1966), que trata sobre la vida y la muerte del filósofo Tomás Moro. Estos problemas de filosofía moral se estudian en El manantial de la doncella (Bergman, 1959). En la sección final del ejercicio se comentan otras películas donde se plantean problemas de moralidad.

Otros filósofos han escrito libros dedicados a plantear un problema filosófico específico a partir del estudio de una película particular. Por ejemplo, Belton (2017) dedicó su trabajo a estudiar la espiral hermenéutica en Vértigo (Hitchcock, 1958), donde se establecen comparaciones puntuales con películas como The Shining (Kubrick, 1980) y la serie Twin Peaks (Lynch). A su vez, otros autores (Bassler, 2017) han estudiado el estado de la filosofía contemporánea a partir de la trilogía de Matrix (Wachowski, 1999; 2003; 2003) o para mostrar los debates sobre la condición humana, la identidad, la conciencia, Dios, la muerte y el tiempo en Blade Runner (Shanahan, 2015).

En todos estos libros $-\mathrm{y}$ muchos otros de naturaleza similar- se puede observar la utilidad pedagógica del cine como herramienta en el proceso de estudiar los problemas centrales del pensamiento filosófico.

\section{Enseñanza de la filosofía a través de directores y géneros}

En esta otra tradición pedagógica se propone la discusión de problemas filosóficos a partir del estudio de directores particulares o géneros como la ciencia ficción, la comedia romántica o el horror. En esta tradición es conocido el trabajo de Singer (2004) sobre la dimensión filosófica en el cine de tres directores, quien estudia la capacidad técnica de Hitchcock, el realismo de Renoir y la integración de estas habilidades en el cine de Welles. El objetivo de ese estudio es mostrar lo que distingue el trabajo de cada director al crear un universo único e irrepetible, que, sin embargo, alcanza un valor universal. 
Esto es muy frecuente en las aproximaciones al estudio de la dimensión filosófica en el trabajo de los directores de cine. Por ejemplo, el reciente trabajo de Pippin (2017) sobre Vértigo de Hitchcock es una reflexión sobre las múltiples dimensiones de la responsabilidad moral que tiene una persona con los otros, en particular en una relación de pareja, donde existen las dimensiones de la identidad real, la identidad percibida por uno mismo, la identidad percibida por el otro y la identidad que cada persona cree que es percibida por el otro. El trabajo del director Terrence Malick tiene especial interés no solo por su calidad cinematográfica, sino por el hecho de que él tiene formación profesional como filósofo, ya que estudió en Harvard con Stanley Cavell. Sus películas han sido objeto de discusiones sobre la representación de la violencia, la relación de los seres humanos con la naturaleza y las relaciones entre luz y obscuridad en películas como La delgada línea roja (1998), El nuevo mundo (2005) y Days of Heaven (1978) (Tucker \& Kendall, 2011). En el caso de Bergman, Livingston (2011) elaboró un estudio sobre los contenidos filosóficos que aparecen con frecuencia en este director, como el intencionalismo, la irracionalidad, la autenticidad y los valores.

El cine de Woody Allen siempre ha despertado el interés de la comunidad filosófica. Ya en el año 2000 la revista Film and Philosophy dedicó un número especial a este director (Lee, 2000). Poco después, en el trabajo coordinado por Conard y Skoble (2004) encontramos aproximaciones a la integridad moral en Manhattan; un análisis kantiano de Crímenes y pecados (1989); el hedonismo en Comedia sexual de una noche de verano (1982); la inautenticidad en Zelig (1983) y el autoconocimiento en La otra mujer (1988).

Por otra parte, algunos filósofos han dirigido una mirada filosófica a géneros del cine clásico, como el horror, la ciencia ficción, el film noir y el cine romántico. En su trabajo sobre el cine de horror, Carroll (2003) propone distinguir entre las tramas de descubrimiento y las tramas protagonizadas por un personaje transgresor. En la presentación a la edición en español, dice: "Al aceptar una pequeña dosis de terror, aspiramos a mejorar el autocontrol sobre nuestras desordenadas emociones, emociones que de hecho nosotros mismos encontramos atemorizantes. [...] El proceso es similar a la inoculación" (p. 17)

La tradición de aproximarse a la filosofía desde las películas de ciencia ficción es muy notable. Mencionemos tan solo la compilación de Sanders (2008) y el estudio de Johnson (2018). En la primera se proponen lecturas filosóficas de películas particulares, como el existencialismo en Frankenstein (Whale, 1931) y las paradojas de los viajes en el tiempo en Terminator (Cameron, 1984) y Doce monos (Gilliam, 1995), y se analiza 2001 (Kubrick, 1968) como una odisea filosófica. En el segundo volumen se estudian en detalle más de treinta películas, como Matrix (Wachowski, 1999) en relación con la libre determinación y el valor del conocimiento-; Interestelar (Nolan, 2014) —en relación con la posibilidad de viajar en el tiempo-; Contacto (Zemeckis, 1997)en relación con el diálogo entre ciencia y religión-, y Gattaca (Niccol, 1994) —en relación con la ética de la reproducción asistida-.

Por su parte, Wolf y Grau (2014) reunieron trabajos sobre la filosofía de la comedia romántica, donde encontramos aproximaciones a la memoria, a la identidad y al deseo en películas como Solaris (Tarkovski, 1972), La sombra de una duda (Hitchcock, 1943) y Una pequeña película de amor (Kieslowski, 1988). El trabajo de Pippin (2012) sobre la filosofía del film noir está centrado en tres películas: Out of the Past (Tourneur, 1947), La dama de 
Shanghai (Welles, 1947) y Scarlett Street (Lang, 1947). En cambio, en la compilación de Conard (2007) sobre la dimensión filosófica del neo-noir - el cine negro producido después de la década de 1980 - se estudian las relaciones entre los antihéroes existencialistas del género y los problemas de identidad y memoria; el nihilismo en el cine de los hermanos Coen y la moral posmoderna en la serie Miami Vice, (1984-1989), entre muchos otros temas de interés filosófico.

En todos estos y otros trabajos similares, se parte de estudiar películas, directores y géneros de cine con el objeto de plantear problemas filosóficos de interés para cualquier espectador, al tiempo que se evita el empleo de un lenguaje técnico y se muestra la relevancia de estas discusiones para la vida cotidiana.

\section{Enseñanza de la ética a través del cine}

La filosofía producida después de la Segunda Guerra Mundial ha estado marcada por el llamado giro estético, vinculado al postestructuralismo (Downing \& Saxton, 2010). En este contexto es posible distinguir dos grandes tendencias en la filosofía moral contemporánea. Por un lado, encontramos a los filósofos que sostienen la apertura al Otro - Agamben, Bajtín, Derrida, Levinas-, y por otra parte los filósofos que defienden la primacía del deseo y, en consecuencia, la primacía del Yo - Badiou, Baudrillard, Bauman, Foucault, Lacan, Zizek-. En todos los casos se plantean problemas de responsabilidad moral, que tienen inevitables consecuencias políticas. Precisamente la teoría feminista ha vinculado estas dimensiones al señalar que lo íntimo siempre es político y tiene consecuencias en la vida social.

Al mismo tiempo, se puede afirmar que un problema central en la teoría del cine es el estudio de la articulación indisociable entre ética y estética, es decir, entre forma y contenido, entre historia y discurso, entre texto y subtexto. En un conocido debate transmitido por televisión en 1959, Godard hizo una declaración filosófica que ha sido muy relevante en los estudios sobre cine: "Le travelling est une question de moral [Un movimiento de cámara es un asunto de moral]" (Downing \& Saxton, 2010, p. 9).

Desde una perspectiva semiótica, esta declaración significa que la forma es el contenido, de tal manera que el empleo de los recursos técnicos del lenguaje cinematográfico tiene consecuencias morales en la experiencia del mundo que se propone a los espectadores.

Además de esta fuerte vinculación entre ética y estética, la estrategia más frecuente para estudiar la dimensión moral de una película de ficción es reconocer cuáles son las decisiones que toma el protagonista frente a los dilemas morales que se le plantean. Un caso paradigmático de decisión moral en el cine clásico se encuentra en el final de Casablanca (Curtiz, 1942), cuando el protagonista decide abordar la avioneta que lo espera y pone el ideal de democracia por encima del amor romántico, lo que en este caso significa poner el deber por encima del deseo. Esto a su vez corresponde a una fuerte tradición judeocristiana de autosacrificio.

En su libro Lo que Sócrates diría a Woody Allen, Juan Antonio Rivera (2003) propone graficar un árbol de decisiones, que corresponde a las decisiones tomadas por el protagonista a lo largo de cualquier largometraje de ficción. Este árbol es muy similar al que se propone en la narratología fílmica, donde se distinguen los núcleos y las catálisis narrativas. A 
su vez, esta serie de decisiones producen una dependencia de la senda ligada al jardín de capacidades, el paisaje rugoso y las órbitas pegajosas o cuencas de atracción

En el cine documental, Nichols $(2001 ; 2010)$ ha propuesto una axiografía que permite estudiar la relación entre los valores y la construcción del espacio fílmico, así como la importancia ideológica de la mirada de la cámara, pues esta establece relaciones específicas entre el observador y lo observado. En términos más generales, Gronstad (2016) propone el estudio de la responsabilidad moral en el empleo del lenguaje cinematográfico para construir una ética general de la forma fílmica. En este contexto, el autor propone seis tesis sobre la imaginación ética en el cine:

1. La forma estética es inherentemente ética.

2. Las imágenes generan su propia ética biovisual.

3. La ética es una hermenéutica.

4. El cine nos muestra otras formas de ser.

5. La imaginación ética se define como apertura, incertidumbre y opacidad.

6. La imaginación ética se opone a las prácticas de la entelequia escópica —entendiendo la entelequia como tomar un caso como si fuera la norma- (pp. 74-99)

Esta visión de la filosofía moral en el cine se desarrolla en el trabajo de Kowalski (2012), que es un libro de texto para estudiantes universitarios de filosofía moral. Este libro contiene doce capítulos sobre temas como el subjetivismo, el relativismo, el contrato social y la amistad. Cada capítulo tiene una extensión de treinta páginas, donde se presenta una sinopsis de la película elegida, un recuadro sobre su contenido moral, una serie de preguntas para debate, y diez páginas de discusión, seguidas por un texto clásico y el comentario sobre otras dos películas donde se plantean problemas morales similares. Por ejemplo, el primer capítulo está dedicado a la retórica y se seleccionó la polémica película Gracias por fumar (Reitman, 2005).

Otro libro de texto dirigido al bachillerato es Seeing the Light (Teays, 2012), donde se exploran diecisiete categorías de la filosofía moral con la discusión de seis películas particulares, lo que da un total de ciento dos películas analizadas. Esto ofrece una gran flexibilidad en el momento de impartir un curso, a partir de los intereses del profesor y los estudiantes, por temas, películas y problemas éticos particulares.

Estos y otros libros de texto tienen como antecedente el monumental trabajo de la historia de la ética elaborado por Rosenstand, cuya primera edición es de 1994. En 2018 se publicó la octava edición, donde no solo se utilizan películas y textos literarios para ilustrar conceptos de la filosofía moral, sino también cómics, fotografías y videojuegos.

En la tradición de los ensayos sobre ética y cine, están los trabajos de Shaw (2012) y Sinnerbrink (2016). En el primero se asocian dieciséis conceptos de la filosofía moral con una película particular, como es el caso de Mar adentro (Amenábar, 2004) y la eutanasia; Citizen Kane (Welles, 1942) y el perspectivismo; o El hombre que mató a Liberty Valance (Ford, 1962) y el contrato social. En el segundo se estudian algunos filósofos de la moral en particular Cavell y Deleuze - y algunos géneros del cine clásico - como el melodrama y el cine de gánsteres-. Este libro ofrece dos útiles apéndices: dieciséis páginas de bibliografía 
comentada por capítulos y una filmografía para la investigación moral con más de 250 títulos de películas clásicas y recientes.

Por último, mencionemos los estudios sobre campos específicos de la filosofía moral: la presencia de la ética del periodismo en el cine (Good, 2008), la ética profesional y de negocios (Skorin, 2019), la ética feminista y del cuidado del otro (Kupfer, 2012), y la ética en el cine de animación para niños (Ward, 2002).

\section{Enseñanza de bioética desde el cine}

Probablemente el campo de la bioética que resulta más inmediato para el espectador común es la ética médica. Mencionemos los trabajos de Colt et al. (2011) y Rosenstand (2018). El primero contiene ochenta ensayos breves - de cinco páginas- sobre un concepto de ética médica asociado a una película particular, que están organizados en ocho áreas de la ética médica: consentimiento informado; profesionalismo; comunicación con el paciente; responsabilidad social; investigación médica; genética y sexualidad; especialidades médicas; y el derecho a morir. Por ejemplo, se analiza la ética de la demencia en Iris (Eyre, 2001), la eutanasia en El paciente inglés (Minghella, 1996) o la discriminación de género en Matar un ruiseñor. El segundo texto estudia diez temas asociados a otras tantas películas y dedica veinte páginas a cada caso, mientras incluye información pertinente acerca de la historia de la medicina. Estos capítulos tratan, por ejemplo, sobre ética pediátrica y la autoridad parental en Lorenzo’s Oil (Miller, 1992), la evaluación de las bondades clínicas y de los daños clínicos en Awakenings (Marshall, 1990) y la ética psiquiátrica vinculada a la competencia profesional en Atrapado sin salida (Forman, 1975).

En Colombia y en México se han publicado recientemente varios volúmenes colectivos sobre los problemas de bioética presentados en el cine. Los publicados en Colombia (Pinto \& Gómez, 2019; Vélez, 2019) reúnen dieciséis ensayos escritos por varios equipos de expertos, donde se tratan problemas bioéticos vinculados a la interrupción del embarazo, las técnicas de procreación humana asistida, epilepsia, tourette, autismo, esclerosis múltiple y problemas que vinculan cuerpo, sexo y cultura. Al final de cada capítulo se incluyen las fichas técnicas de las películas discutidas y su pertinencia para un seminario temático. El volumen publicado en México (García, 2011) reúne veinticinco ensayos breves sobre temas similares, además de incluir una filmografía integrada por sesenta películas de ficción sobre problemas de bioética.

El otro gran terreno de la investigación bioética en el cine, sin duda el más trascendente, es la ética ambiental. A partir de la década de 1990, se han publicado ya varias docenas de libros sobre este apasionante terreno del cine contemporáneo, de los cuales mencionaré algunos de los más relevantes. Aquí es necesario señalar el cambio de paradigma que ha significado pasar de películas como Erin Brokovich (Soderbergh, 2000), en las que se presenta una agenda ecológica como trasfondo narrativo al servicio del entretenimiento, a películas como Una verdad incómoda (Guggenheim, 2006), cuya intención es provocar en los espectadores la toma de conciencia para sacar adelante alguna forma de activismo ecológico a favor de la naturaleza.

Esto último se señala en el libro colectivo de Willoquet (2010) y es muy próximo al espíritu del trabajo de Vaughan (2019), donde se revelan los monumentales costos en 
el consumo de agua y otros recursos ambientales de la producción, la distribución y el consumo de cine en la cultura digital contemporánea.

Otros libros sobre la ética ambiental son los de Brereton (2005; 2016), Ingram (2010), Moore (2017), Narine (2015) y O’Brien (2018), así como los coordinados por Gurr (2015), Murray \& Heuman (2009) y Rust et al. (2013), donde ya se habla de ecofilosofía, ecocine y el cine como máquina antrobiogeomórfica, donde se articulan problemas de raza, de clase, de género y de sexualidad en relación con la naturaleza, lo mismo en westerns que en road movies, ciencia ficción, melodrama y thrillers. En estos trabajos ya se habla de una ecología profunda, que es una ética basada en sistemas de creencia no antropocéntricos (Brereton, 2016).

En México, estos problemas son analizados en el Manual de cine y ética para el siglo XXI (Ramírez, 2009) y en el estudio de González de Arce (2019) sobre el cine mexicano clásico desde la perspectiva ecocrítica.

\section{Política y filosofía a través del cine}

Un grupo importante de filósofos dedica su trabajo a reflexionar sobre la dimensión política del mundo actual y su representación en la ficción hollywoodense y en el cine independiente. Por ejemplo, Baumbach (2019) estudia estos problemas desde la perspectiva de Rancière, Deleuze y Benjamin. Por su parte, Herzberg (2019) y Mazierska \& Kristensen (2014; 2015) estudian el cine elaborado desde una perspectiva marxista, como el cine militante latinoamericano de la década de 1960, La hora de los hornos (Getino \& Solanas, 1968), y el cine de Godard y de Chris Marker.

Wartenberg (1999) ha identificado películas románticas en las que se pone en evidencia una postura política, como en Sucedió una noche (Capra, 1934), Adivina quién viene a cenar (Kramer, 1967) y Mujer bonita (Marshall, 1990). Hilliard (2009) estudia las películas que han denunciado problemas de pobreza, de racismo, de sexismo y de guerra. Glover y Tagliavina (2013) han reunido ensayos sobre la discusión de problemas sociales en el salón de clase con el empleo de recursos como novela gráfica, fotografía, literatura, música, teatro, cine, televisión y las redes sociales.

En todos estos casos se trata de convertir la reflexión filosófica en una práctica política en el interior del aula de cine, lo que con frecuencia lleva pasar de la denuncia al activismo educativo.

\section{Comentarios generales}

En muchos de los libros comentados se logra un equilibrio entre una perspectiva panorámica - overview - y el análisis de películas o secuencias plano por plano - close reading-. De esa manera se atiende su naturaleza estética, que es lo que las hace atractivas y estimulantes - la forma fílmica -, sin dejar de lado los contenidos de filosofía moral que las hace relevantes - el subtexto filosófico-. Precisamente esta doble integración es lo que hace del cine un terreno intensamente pedagógico.

Una herramienta muy útil para lograr este objetivo es la creación de un sitio en la red, donde cualquier persona tiene acceso gratuito a clips de cine seleccionados para abrir la 
discusión sobre problemas de ética. Wartenberg creó un sitio ${ }^{1}$ orientado a los estudiantes de secundaria y bachillerato, donde se encuentran clips vinculados con problemas como el bullying, la presión social de los compañeros y la ética ambiental.

Por su parte, son muy conocidos los documentales donde Zizek comenta desde una perspectiva lacaniana escenas canónicas del cine de Hitchcock, de Lynch, de Kubrick y de muchos otros directores, en Guía del cine para pervertidos (Phiennes, 2006) y Guía ideológica para pervertidos (Phiennes, 2012).

Es notable el hecho de que algunos autores hayan elaborado libros de texto sobre la ética dirigidos a estudiantes universitarios (Kowalski, 2012; Ramírez, 2009; Peña, 2014; Rosenstand, 2018) o para el bachillerato (Brunet, 2003; Teays, 2012), incluyendo numerosos autores en México - por ejemplo, Ojeda et al. (2007) y en España -Güell y Rendón (2008), entre otros-.

Algunos autores estudian áreas específicas de la filosofía a través del cine, como la lógica (Polette, 2005), la metafísica (Fraser \& Vernon, 2000; Marsh, 2007; Rodríguez, 2002), la ontología (Niemiec \& Wedding, 2014), la epistemología (Ehrat, 2015) y la estética (Thomson-Jones, 2008; Tierno, 2002; Isaacs, 2008; Pezzela, 2004). Sin embargo, es evidente que la mayor parte de los filósofos que utilizan el cine como herramienta para estudiar filosofía se centran en los problemas siempre actuales de la ética.

Otros investigadores estudian la filosofía de autores específicos en relación con el cine. Así, encontramos estudios sobre la filosofía del cine en Agamben (Gustafsson \& Gronstad, 2014), en Badiou (Ling, 2011), en Cavell (Read \& Goodenough, 2005; Rothman \& Keane, 2000, Shaw, 2012), en Deleuze (Bogue, 2003; Colman, 2011; Marrati, 2003; Pisters, 2003, 2015; Redner, 2011, Torres, 2012), en Kristeva (Goodnow, 2010), en Lacan (Zizek, 1994), en Levinas (Girgus, 2010; Hole, 2016), en Peirce (Ehrat, 2015), en Rancière (Casas \& Farfán, 2014), en Wittgenstein (Szabados \& Stojanova, 2011) y en Zizek (Flisfeder, 2012). Otros filósofos adoptan una perspectiva más general y diversa al aproximarse a la filosofía y el cine, como es el caso de Cabrera (2015), Cox y Levine (2012), Falzon (2005), Gilmore (2005), Herzogenrath (2017), Phillips (2008), Shamir (2016), Shaw (2008), Sinnerbrink (2011), Wartenberg (2007). Y en algunos volúmenes colectivos se ofrecen aproximaciones muy diversas al diálogo entre cine y filosofía, como ocurre en las compilaciones de Livingston \& Plantinga (2009), Thomson-Jones (2016) y Carroll, Summa \& Loht (2019).

Al estudiar el costo ambiental que tiene la producción de cada película en términos de consumo de agua y otros recursos ambientales, el trabajo de Vaughan (2019) señala la necesidad de realizar un cambio de paradigma en el estudio de la filosofía a través del cine, en el estudio del cine desde una perspectiva filosófica y en los estudios filosóficos en general:

La cultura de las pantallas es una fuerza social dinámica en la que se trabaja al mismo tiempo en la estética y la retórica, la atracción visual y las estrategias para elaborar mensajes cognitivos y en la producción de un impacto ambiental significativo [...] Las humanidades ambientales deberán comprometerse con perspectivas empíricas como la sociología, la psicología y la antropología. (p. 17)

Como se ha podido observar en este recorrido bibliográfico, la tradición filosófica

1 http://whatsthebigideaprogram.com/ 
continental tiene un carácter nomotético - normativo-y utiliza el cine como un estímulo, un soporte y un referente para la creación del pensamiento filosófico, el cual es elaborado, con frecuencia, en un lenguaje técnico, dirigido al lector especializado en filosofía. En contraste, la tradición filosófica analítica tiene un carácter casuístico y utiliza las herramientas de la filosofía para entender los contenidos filosóficos y las connotaciones de la forma fílmica que pueden ser encontradas en cualquier película. Esta argumentación es elaborada, con frecuencia, con fines pedagógicos y, por eso mismo, en un lenguaje accesible y dirigido al lector no especializado. En síntesis, la tradición continental es altamente especializada y pone al cine al servicio de la filosofía, mientras que la tradición analítica tiene una vocación pedagógica y pone la filosofía al servicio de la cinefilia.

Es evidente que el diálogo entre el cine y la filosofía es intenso, productivo y relevante para la vida individual y colectiva. Con frecuencia, establece diálogos igualmente productivos con las otras disciplinas universitarias.

\section{Referencias}

Allen, W. (1978). (Director). Interiores. [Película]. United Artists

Allen, W. (1979). (Director). Manhattan. [Película]. United Artists

Allen, W. (1982). (Director). Comedia sexual de una noche de verano. [Película]. Orion Pictures

Allen, W. (1983). (Director). Zelig. [Película]. Orion Pictures, Warner Bros.

Allen, W. (1985). (Director). La rosa púrpura del Cairo. [Película]. Orion Pictures

Allen, W. (1988). (Director). La otra mujer. [Película]. Orion Pictures

Allen, W. (1989). (Director). Crímenes y pecados. [Película]. Jack Rollins \& Charles H. Joffe Production.

Almodóvar, P. (2002). (Director). Hable con ella. [Película]. El Deseo S.A.

Amenábar, A. (2004). (Director). Mar adentro. [Película]. Sogecine Himenóptero.

Annaud, J.-J. (1984). (Director). El nombre de la rosa. [Película]. Constantin Film.

Bassler, O. (2017). Diagnosing Contemporary Philosophy with The Matrix Movies. Palgrave Macmillan.

Baumbach, N. (2019). Cinema / Politics / Philosophy. Columbia University Press.

Beaney, M. (2017). Analytic Philosophy. A Very Short Introduction. Oxford University Press.

Belton, R. (2017). Alfred Hitchcock's Vertigo and the Hermeneutic Spiral. Palgrave Macmillan.

Bernini, E., Gaetano, R. \& Dottorini, D. (2015). Cine y filosofía. Las entrevistas de Fata Morgana. El Cuenco de Plata.

Bogue, R. (2003). Deleuze on Cinema. Routledge.

Brereton, P. (2005). Hollywood Utopia. Ecology in Contemporary American Cinema. 
Intellect Books.

Brereton, P. (2016). Environmental Ethics and Film. Routledge.

Brown, R., Decker, K., \& Irwin, W. (2009). Terminator and Philosophy: I'll be Back, Therefore I Am. The Blackwell Philosophy and Popular Culture Series. John Wiley and Sons.

Brunet, G. (2003). Ética y narración. Édere.

Cabrera, J. (2015). Cine: 100 años de filosofía. Una introducción a la filosofía a través del análisis de películas (2a ed.). Gedisa.

Cameron, J. (1984). (Director). Terminator. [Película]. Hemdale Film.

Capra, F. (1934). (Director). Sucedió una noche. [Película]. Columbia Pictures.

Carroll, N. (2003). Engaging the Moving Image. Yale University Press.

Carroll, N., Summa di, L. \& Loht, S. (2019). The Palgrave Handbook of the Philosophy of Film and Motion Pictures. Palgrave Macmillan.

Casas, A. \& Farfán, L. (2014). Jacques Rancière. En los bordes del cine. UNAM.

Chateau, D. (2005). Cine y filosofía. Colihue.

Colman, F. (2009). Film, Theory and Philosophy. The Key Thinkers. McGill-Queen's University Press.

Colman, F. (2011). Deleuze \& Cinema. The Film Concepts. Berg.

Colt, H., Quadrelli, S. \& Friedman, L. (2011). The Picture of Health. Medical Ethics and the Movies. Oxford University Press.

Conard, M. \& Skoble, A. (2004). Woody Allen and Philosophy. You Mean My Whole Fallacy Is Wrong? Open Court.

Conard, M. (2007). The Philosophy of Neo-Noir. The University Press of Kentucky.

Coppola, F. F. (1979). (Director). Apocalypse Now. [Película]. Zoetrope Studios.

Cox, D. \& Levine, M. (2012). Thinking through Film. Doing Philosophy, Watching Movies. Wiley-Blackwell.

Curtiz, M. (1942). (Director). Casablanca. [Película]. Warner Bros.

D’Agostini, F. (2000). Analíticos y continentales. Guía de la filosofía de los últimos treinta años. Cátedra. Edición original: Analitici e continentali (1997). Edizione CDE.

Dainton, B. \& Robinson, H., eds. (2014). The Bloomsbury Companion to Analytic Philosophy. Bloomsbury

Davies, D., ed. (2009). The Thin Red Line. Philosophers on Film. Routledge.

Deleuze, G. (1972). Mil mesetas. Capitalismo y esquizofrenia. Pre-Textos.

Deleuze, G. (1981). Cine I. Bergson y las imágenes. Cactus.

Deleuze, G. (1983). La imagen-movimiento. Estudios sobre cine 1. Paidós. 
Deleuze, G. (1985). La imagen-tiempo. Estudios sobre cine II. Paidós.

Deleuze, G. (1989). El pliegue. Leibniz y el Barroco. Paidós

Deleuze, G. (2002). Diferencia y repetición. Amorrortu.

Didi-Huberman, G. (2016). Pueblos en lágrimas, pueblos en armas. Contracampo.

Downing, L. \& Saxton, L. (2010). Film and Ethics. Foreclosed Encounters. Routledge.

Eaton, A., ed. (2009). Talk to Her. Philosophes on Film. Routledge.

Ehrat, J. (2015). Cinema and Semiotic. Peirce and Film Aesthetics, Narrative and Representation. University of Toronto Press.

Esquenazi, J. (2017). L’analyse de film avec Deleuze. CNRS.

Eyre. R. (2001). (Director). Iris. [Película]. Miramax.

Falzon, C. (2005). La filosofía va al cine. Una introducción a la filosofía. Alianza.

Ferrer, A., García, X., Hernández, F. \& Lerma, B. (2006). Primum videre, deinde philosophari. Una Historia de la Filosofía a través del cine. Fondaments.

Ford, J. (1962). (Director). El hombre que mató a Liberty Valance. [Película]. Paramount Pictures

Fraser, P. \& Vernon, E. (2000). Reviewing the Movies. A Christian Response to Contemporary Film. Crossway.

García, G. (2011). Cine y bioética. Asociación de Bioética y Derechos Humanos Netemachillizpan

Getino, O. \& Solanas, F. (1968). (Directores). La hora de los hornos. [Película].

Gilliam, T. (1975). (Director). Los caballeros de la mesa cuadrada. [Película]. Michael White Productions.

Gilliam, T. (1995). (Director). Doce monos. [Película]. Universal Pictures.

Gilmore, A. (2005). Doing Philosophy at the Movies. State University of New York.

Girgus, S. (2010). Levinas and the Cinema of Redemption. Time, Ethics, and the Feminine. Columbia University Press.

Glock, H.-J. (2008). What is Analytic Philosophy? Cambridge University Press.

Glover, R. \& Tagliavina, D. (2013). Teaching Politics Beyond the Book. Bloomsbury.

Gondry, M. (2004). (Director). Eterno resplandor de una mente sin recuerdos. [Película]. Anonymous Content.

González de Arce, R. (2019). El viaje del cine mexicano de ficción hacia la conciencia ecológica. Imaginarios de la naturaleza, ecoutopías y ética ambiental en la pantalla [Tesis de Maestría]. Universidad Iberoamericana. Tesis no publicada

Good, H. (2008). Journalism Ethics Goes to the Movies. Rowman and Littlefield.

Goodnow, K. (2010). Kristeva on Focus. From Theory to Film Analysis. Berghan Books. 
Grau, C., ed. (2009). Eternal Sunshine of the Spotless Mind. Philosophers on Film. Routledge.

Grodal, T. (1997). Moving Pictures. A New Theory of Film Genres, Feelings, and Cognition. Clarendon Press.

Gronstad, A. (2016). Film and the Ethical Imagination. Palgrave Macmillan.

Güell, M. \& Redón, J. (2008). Filosofía y ciudadanía. $1^{\circ}$ de Bachillerato. Octaedro.

Guggenheim, D. (2006). (Director). Una verdad incómoda. [Película]. Lawrence Bender Productions.

Gurr, B. (2015). Race, Gender, and Sexuality in Post-Apocalyptic TV and Film. Palgrave Macmillan.

Gustafsson, H. \& Gronstad, A. (2014). Cinema and Agamben. Ethics, Biopolitics and the Moving Image. Bloomsbury.

Herzberg, B. (2009). The Left Side of the Screen. Commitment and Left-Wing Ideology in Hollywood, 1929-2009. McFarland.

Herzogenrath, B. (2017). Film as Philosophy. University of Minnesota Press.

Hilliard, R. (2009). Hollywood Speaks Out. Pictures that Dared to Protest Real World Issues. Wiley-Blackwell.

Hitchcock, A. (1943). (Director). La sombra de una duda. [Película]. Universal Pictures.

Hitchcock, A. (1954). (Director). Rear Window (La ventana indiscreta). [Película]. Patron Inc.

Hitchcock, A. (1958). (Director). Vértigo. [Película]. Alfred J. Hitchcock Productions.

Hole, K. (2016). Towards a Feminist Cinematic Ethics. Claire Denis, Emmanuel Levinas and Jean-Luc Nancy. Edinburgh University Press.

Ingram, D. (2010). Environmentalism and Hollywood Cinema. University of Exeter Press.

Irwin, W. \& Jacoby, H. (2009). La filosofía de House. Todos mienten. Selector.

Irwin, W., Conard, M. \& Skoble, A. (2009). Los Simpson y la filosofía. Blackie books.

Isaacs, B. (2008). Toward a New Film Aesthetic. Continuum.

Jarvie, I. (1987). Philosophy of the Film. Epistemology, Ontology, Aesthetics. Routledge. Traducción al español: Filosofía del cine (2011). Síntesis.

Jeunet, J.-P. (2001). (Director). Amélie. [Película]. Claudie Ossard Productions.

Johnson, D. (2018). Sci-Phi. Science Fiction as Philosophy. Course Guidebook. The Great Courses.

Kania, A., ed. (2009). Memento. Philosophers on Film. Routledge.

Kieslowski, K. (1988). (Director). Una pequeña película de amor. [Película]. TOR film studio.

Kowalski, D. (2012). Moral Theory at the Movies. An Introduction to Ethics. Rowman \& 
Littlefield.

Kramer, S. (1967). (Director). Adivina quién viene a cenar. [Película]. Columbia Pictures.

Kubrick, S. (1964). (Director). Cómo aprendí a amar la bomba. [Película]. Hawk Films.

Kubrick, S. (1968). (Director). 2001.Odisea del espacio. [Película]. Metro-Goldwyn-Mayer.

Kubrick, S. (1980). (Director). The Shining (El resplandor). [Película]. Warner Bros. Pictures.

Kupfer, J. (2012): Feminist Ethics in Film. Reconfiguring Care through Cinema. Intellect.

Lang, F. (1947). (Director). Scarlett Street (Perdición). [Película].

Lee, S. (2000). Film and Philosophy. Special Issue on Woody Allen. Society for the Philosophical Study of the Visual Arts.

Ling, A. (2011). Badiou and Cinema. Edinburgh University Press.

Livingston, P. \& Plantinga, C., eds. (2009). The Routledge Companion to Philosophy and Film. Routledge

Livingston, P. (2011). Cinema, Philosophy, Bergman. On Film as Philosophy. Oxford University Press.

Lynch, D. (2001). (Director). Mulholland Drive. [Película]. Les Films Alain Sarde.

Malick, T. (1978). (Director). Days of Heaven. [Película]. Paramount Pictures.

Malick, T. (1998). (Director). La delgada línea roja. [Película]. 20th Century Fox.

Malick, T. (2005). (Director). El nuevo mundo. [Película]. New Line Cinema.

Marrati, P. (2003). Gilles Deleuze. Cine y filosofía. Claves.

Marsh, C. (2007). Theology Goes to the Movies. An Introduction to Critical Christian Thinking. Routledge.

Marshall, P. (1990). (Directora). Awakenings (Despertares). [Película]. Lasker/Parkes Productions.

Mazierska, E. \& Kristensen, L. (2014). Marx at the Movies. Revisiting History, Theory and Practice. Palgrave Macmillan.

Mazierska, E. \& Kristensen, L. (2015). Marxism and Film Activism. Screening Alternative Worlds. Bertghahn Books.

Miller, G. (1992). (Director). Lorenzo’s Oil. [Película]. Universal Pictures.

Minghella, A. (1996). (Director). El paciente inglés. [Película]. Miramax.

Moore, E. (2017). Landscape and Environment in Hollywood Film. The Green Machine.

Mullarkey, J. \& Lord, B. (2009). The Continuum Companion to Continental Philosophy. Continuum.

Mullarkey, J. (2009). Refraction of Reality. Philosophy and the Moving Image. Palgrave Macmillan. 
Mulligan, R. (1962). (Director). Matar un ruiseñor. [Película]. Universal Pictures.

Murray, R. \& Heuman, J. (2009). Ecology and Popular Film. State University of New York Press.

Narine, A, (2015). Eco-Trauma Cinema. Routledge.

Niccol, A. (1994). (Director). Gattaca. [Película]. Columbia Pictures.

Nichols, B. (2001). Introduction to Documentary. Indiana University Press.

Nichols, B. (2010). Engaging Cinema. An Introduction to Film Studies. W. W. Norton.

Niemiec, R. \& Wedding, D. (2014). Positive Psychology at the Movies. Hogrefe.

Nolan, C. (2000). (Director). Memento. [Película]. Newmarket Films.

Nolan, C. (2014). (Director). Interestelar. [Película]. Legendary Pictures.

O’Brien, A. (2018). Film and the Natural Environment. Wallflower.

Ojeda, M., Arizmendi, P. \& Rivero, E. (2007). Ética. Una visión global de la condición humana. Pearson.

Peña, B. (2014). La transmisión de valores a través del lenguaje cinematográfico. Dykinson.

Pezzela, M. (2004). Estética del cine. La Balsa de la Medusa.

Phiennes, S. (2006). (Director). Guía del cine para pervertidos. [Película]. Coproducción Reino Unido-Austria-Países Bajos (Holanda).

Phiennes, S. (2012). (Director). Guía ideológica para pervertidos. [Película]. Coproducción Reino Unido-Irlanda.

Phillips, J. (2008). Cinematic Thinking. Philosophical Approaches to the New Cinema. Stanford University Press.

Pinto, B. J. \& Gómez, A. I., eds. (2019). Conflictos y paradojas: cine y bioética en el inicio de la vida. Universidad del Rosario.

Pippin, R. (2012). Fatalism in American Film Noir. Some Cinematic Philosophy. The University of Virginia Press.

Pippin, R. (2017). The Philosophical Hitchcock. Vertigo and the Anxieties of Unknowingness. The University of Chicago Press.

Pisters, P. (2003). The Matrix of Visual Culture. Working with Deleuze in Film Theory. Stanford University Press.

Pisters, P. (2015). The Neuro-Image. A Deleuzian Film-Philosophy of Digital Screen Culture. Stanford University Press.

Polette, N. (2005). Teaching Thinking Skills with Fairy Tales and Fantasy. Teacher Ideas Press.

Ramírez, R. (2009). Manual de cine y ética para el siglo XXI. Cineteca Nacional; Universidad Pedagógica Nacional; Universidad Anáhuac; Universidad de la Sustentabilidad. 
Rancière, J. (2001). La fábula cinematográfica. Titivilius.

Rancière, J. (2010). El espectador emancipado. Manantial.

Rancière, J. (2011). Las distancias del cine. Manantial.

Rancière, J. (2014). En los bordes del cine. UNAM.

Read, R. \& Goodenough, J., (2005). Film as Philosophy. Essays on Cinema after Wittgenstein and Cavell. Palgrave Macmillan.

Redner, G. (2011). Deleuze and Film Music. Building a Methodological Bridge between Film Theory and Music. Intellect.

Reitman, J. (2005). (Director). Gracias por fumar. [Película]. Room 9 Entertainment.

Rivera, J. A. (2003). Lo que Sócrates diría a Woody Allen. Cine y filosofía. Espasa Calpe

Rodríguez, M. (2002). Cine y cristianismo. Universidad Católica San Antonio.

Rosenstand, N. (2018). The Moral of the Story. An Introduction to Ethics ( $8^{\mathrm{a}}$ ed.). McGrawHill.

Rosset, C. (2010). Reflexiones sobre cine. El Cuenco de Plata.

Rothman, W. \& Keane, M. (2000). Reading Cavell's The World Viewed. A Philosophical Perspective on Film. Wayne State University Press.

Rust, S., Monani, S. \& Cubbitt, S. (2013). Ecocinema Theory and Practice. American Film Institute.

Sanders, S. (2008). The Philosophy of Science Fiction Film. The University Press of Kentucky.

Schrift, A. D., ed. (2010). The History of Continental Philosophy. Volumes 1-8. The University of Chicago Press.

Scott, R. (1982). (Director). Blade Runner. [Película]. The Ladd Company.

Shamir, T. (2016). Cinematic Philosophy. Palgrave Macmillan.

Shanahan, T. (2015). Philosophy and Blade Runner. Palgrave Macmillan.

Shaw, D. (2008). Film and Philosophy. Wallflower.

Shaw, D. (2012). Morality and the Movies. Reading Ethics through Film. Continuum.

Singer, I. (2004). Three Philosophical Filmmakers. Hitchcock, Welles, Renoir. The MIT Press.

Sinnerbrink, R. (2011). New Philosophies of Film. Thinking Images. Continuum.

Sinnerbrink, R. (2016). Cinematic Ethics. Exploring Ethical Experience through Film. Routledge.

Skorin, J. (2019). Professional and Business Ethics through Film. The Allure of Cinematic Presentation and Cultural Thinking. Palgrave Macmillan.

Soderbergh, S. (2000). (Director). Erin Brockovich. [Película]. Jersey Films.

Solomon, R. \& Sherman, D. (2009). The Blackwell Guide to Continental Philosophy. 
Blackwell.

Spielberg, S. (1975). (Director). Jaws (Tiburón). [Película]. Universal Pictures.

Spielberg, S. (1993). (Director). Parque Jurásico. [Película]. Amblin Entertainment.

Szabados, B. \& Stojanova, C. (2011). Wittgenstein at the Movies. Cinematic Investigations. Rowman \& Littlefield.

Tarkovski, A. (1972). (Director). Solaris. [Película]. Mosfilm.

Teays, W. (2012). Seeing the Light. Exploring Ethics through the Movies. Wiley-Blackwell.

Thomson-Jones, K. (2008). Aesthetics \& Film. Continuum.

Thomson-Jones, K., ed. (2016). Current Controversies in Philosophy of Film. Routledge.

Tierno, M. (2002). Aristotle's Poetics for Screenwriters. Storytelling Secrets from the Greatest Mind in Western Civilization. Hyperion.

Torres, S. (2012). (Montajes). Entre filosofía y cine. Torres Asociados.

Tourneur, J. (1947). (Director). Out of the Past. [Película]. RKO Radio Pictures

Tucker, T. \& Kendall, S. (2011). Terrence Malick: Film and Philosophy. Continuum.

Vaughan, H. (2019). Hollywood's Dirtiest Secret. The Hidden Environmental Costs of the Movies. Columbia University Press.

Vélez, A., ed. (2019): Neurociencia y cine. Universidad del Rosario.

Wachowski, L. \& L. (1999). (Directoras). Matrix. [Película]. Village Roadshow Pictures.

Wachowski, L. \& L. (2003). (Directoras). Matrix Reloaded. [Película]. Village Roadshow Pictures.

Wachowski, L. \& L. (2003). (Directoras). Matrix Revolutions. [Película]. Village Roadshow Pictures.

Ward, A. (2002). Mouse Morality. The Rhetoric of Disney Animated Film. University of Texas Press.

Wartenberg, T. (1999). Unlikely Couples. Movie Romance as Social Criticism. Worllview Press.

Wartenberg, T. (2007). Thinking on Screen. Film as Philosophy. Routledge,

Welles, O. (1942). (Director). Citizen Kane. [Película]. Mercury Productions.

Welles, O. (1947). (Director). La dama de Shanghai. [Película]. Columbia Pictures.

Whale, J. (1931). (Director). Dr. Frankenstein. [Película]. Universal Pictures.

Willoquet, P, (2010). Framing the World. Explorations in Ecocriticism and Film. University of Virginia Press.

Wolf, S. \& Grau, C. (2014). Understanding Love. Philosophy, Film, and Fiction. Oxford University Press.

Zemeckis, R. (1997). (Director). Contacto. [Película]. Warner Bros. 
Zimmermann, F. (1966). (Director). Un hombre para la eternidad. [Película]. Highland Films.

Zizek, S. (1994). Todo lo que usted siempre quiso saber sobre Lacan y nunca se atrevió a preguntarle a Hitchcock. Manantial.

\section{Bibliografía}

A continuación, se relacionan otros libros sobre cine y filosofía que pueden ser consultados.

Bagget, D., \& Drumin, W., eds. (2007). Hitchcock and Philosophy. Dial M for Metaphysics. Popular Culture and Philosophy. Open Court.

Carroll, N. (1998). Una filosofía del arte de masas. La Balsa de la Medusa.

Carroll, N. (2008). The Philosophy of Motion Pictures. Blackwell.

Cavell, S. (1979). The World Viewed. Reflections on the Ontology of Film. Harvard University Press.

Cavell, S. (1981). Pursuits of Happiness. The Hollywood Comedy of Remarriage. Harvard University Press. Traducción al español: La búsqueda de la felicidad. La comedia de enredo matrimonial en Hollywood (1999). Paidós

Cavell, S. (1996). Más allá de las lágrimas. La Balsa de la Medusa.

Cavell, S. (2008). El cine ¿puede hacernos mejores? Katz.

Chritchley, S. (2001). Continental Philosophy. A Very Short Introduction. Oxford University Press.

Frampton, D. (2006). Filmosophy. Wallflower.

Giannopoulou, Z., ed. (2013). Mulholland Drive. Philosophers on Film. Routledge.

Grodal, T. (2009). Embodied Visions. Evolution, Emotion, Culture and Film. Oxford University Press.

Grodal, T. (2019). El flujo PECMA: una teoría general de la experiencia del cine. En J. Conde (Ed.), Semióticas del cine y del audiovisual (pp. 23-47). Universidad de Bogotá Jorge Tadeo Lozano.

Makkai, K., ed. (2013). Vertigo. Philosophers on Film. Routledge.

Rivera, J. A. (2005). Carta abierta de Woody Allen a Platón. Espasa Calpe.

Singer, I. (2007). Ingmar Bergman, Cinematic Philosopher. Reflections on His Creativity. The MIT Press.

Zizek, S. (2001). The Fright of Real Tears. Krzysztof Kieslowski between Theory and PostTheory. British Film Institute. Traducción al español por Sergio Aguilar: El espanto de lágrimas reales (2020). Universidad Iberoamericana. 